is there sufficient evidence of regression to worry about its implications? There is no regression effect in our $60-5$ group (see Fig. 1). (Their strongest regression effect was in the 60-5 group.) Notice, also, that our $60-5$ group was faster on the first postshift trial than on subsequent trials. Panksepp et al attribute significance to the fact that their $60-5$ group was slower on this trial than on subsequent trials. Our $5-60$ group shows some regression, but it does not approach the preshift baseline. Seven of our eight $S$ s were significantly above (60-5 group) or below (5-60 group) the preshift baseline on the final day of postshift testing. (Our study had 100 postshift trials vs 75 in theirs) and the eighth $S$ showed no regression-it did not show the effect at all (see Fig. 2). In the Panksepp et al study, 5 of 10 (or 6 of 10 -it is difficult to classify their S 6) showed a significant tendency to regress (as gauged by comparing the last postshift day against the first postshift day). In our study 4 of 8 showed such a tendency. Thus, taking the two studies together, about half the Ss showed any significant tendency to regress, let alone a regression to the baseline. One would expect several Ss to show "regression" by chance alone, since in both studies, under all conditions (pre- and postshift), Ss showed significant bidirectional day-to-day changes in performance (see Fig. 2). Thus, there was a decent chance of observing a "regression" when one compared any one day against any other day. We are not sure what the appropriate statistical test would be here, but it seems clear that not only are the implications of the effect unclear, it is unclear that there is an effect.

Finally, it is curious that Panksepp et al should cite in support of their argument Scott's (1967) report that there was no overnight decrement in well trained $S$ s. Scott's data contradict their own. Using 15 trials per day, Scott found no warm-up effect on the 5th, 6th, and 7 th day of training. After at least 6 days of training (and apparently more), at 15 trials a day, Panksepp et al not only report a strong warm-up effect, they refer to it as an effect which is "often found in animals running for ESB." That Scott did not find a warm-up effect is readily explained by a drive (or arousal) decay hypothesis: He used a 15-min intertrial interval.

\section{REFERENCES}

DEUTSCH, J, A. Behavioral measurement of the neural refractory period and its application to intracranial self-stimulation. Journal of Comparative \& Physiological Psychology, 1964, 58, 1-9.

DEUTSCH, J. A., ADAMS, D. W., \& METZNER, R. J. Choice of intracranial stimulation as a function of delay between stimulation and strength of competing drive. Journal of Comparative \& Physiological Psy chology, 1964, $57,241-243$.
GALLISTEL, C. R. Intracranial stimulation and natural rewards: Differential effects of trial spacing. Psychonomic Science, 1967, 9, 163-168.

PANKSEPP, J., GANDELMAN, R., \& TROWILL, $J$. The effect of intertrial interval on running performance for ESB. Psychonomic Science, $1968,13,135-136$.

SCOTT, J. W. Brain stimulation reinforcement with distributed practice: Effects of electrode locus, previous experience and stimulus intensity. Journal of Comparative \& Phy siological Psychology, 1967, 63, 175-183. NOTE

1. Gallistel, C. R., Rolls, E., \& Greene, D. Neuronal function inferred by behavioral and electrophysiological measurement of the neural refractory period. Submitted to Science, 1969.

\title{
Reply to Gallistel
}

JAAK PANKSEPP, RONALD GANDELMAN, and JAY A. TROWILL, University of Massachusetts, Amherst, Mass. 01002

Gallistel's comment (see this issue) on our study, "The effect of intertrial interval on running performance for ESB" (Panksepp, Gandelman, \& Trowill, 1968), includes some provocative data and terminological comments about the interpretation of performance maintained by rewarding electrical stimulation of the brain (ESB). Gallistel originally reported (1967) that increasing intertrial interval (ITI) from 5 to $60 \mathrm{sec}$ increases nunning speed of waterdeprived rats responding for water but decreases running speed when animals are responding for ESB. Our replication of this procedure confirmed Gatlistel's results. However, a trial-by-trial analysis of our data revealed that many $S$ s showed a regression of running speeds toward the preshift baseline across postshift days. Thus, the first postshift trial emphasized by Gallistel may not have been representative of subsequent postshift trials. In fact, it was our contention that such an incomplete presentation of data could be misleading.

Gallistel's comment consists of several arguments and data which he interprets as supporting the "drive-decay" position of Deutsch \& Howarth (1963) and of Gallistel (1964). Since Gallistel has now provided session-by-session and trial-by-trial data which can be directly compared to our own data, we are in a position to reply to his comments.

Before dealing with the specific comments of Gallistel, attention should be drawn to the over- and underresponding during postshift trials as compared to preshift levels (Fig. 1 of Gallistel's article). It is tempting to suggest a similarity between these overreactions and the positive (elation) and negative (depression) contrast effects found with upshifts and downshifts of ESB current intensity (Panksepp \& Trowill, 1969). Although ITI shifts have not yet been clearly defined as incentive manipulations, we propose that the similarity of effects following such shifts warrants this consideration.

In our original article we felt reticent about proposing that ITI shifts are, in fact, incentive shifts. Now, having had the opportunity to also view Gallistel's data, the close similarity between his data and our own, especially in the 5- to 60-sec group, buttresses this supposition. If ITI is an incentive variable, then it would be reasonable to expect that the higher incentive (5-sec ITI) should show more rapid performance than the lower-incentive condition, as is so commonly found in varying the amount of reward. Viewing ITI as an incentive variable, shifts in ITI should lead to changes in performance to the appropriate incentive level after an initial and transitory contrast effect, rather than to the preshift baseline. Such a contrast and complete return to appropriate baseline is strikingly clear in the 5-60 group (Fig. 1Gallistel's data). Gallistel suggests that the regression of postshift performance may merely represent spurious session-to-session fluctuations. However, the regression, rather than fluctuating, shows systematic trends toward the preshift baseline. The regression is not minimal as Gallistel implies, especially in the 5-60 animals, six of nine of which, in the two experiments, showed significant regression to baseline. In all, $50 \%$ of the Ss showed significant regression--an impressive percentage considering that if no true effect is present in the data only one $S$ out of 20 should reach the .05 level by chance alone. Furthermore, in all cases except one (Gallistel's S-27) regression occurred in the direction predicted by incentive contrast. It thus seems unlikely that the regression is either trivial or a mere reflection of random fluctuations.

Gallistel suggests that the difference between his drive interpretation and our incentive-motivation interpretation may rest purely on terminological grounds. Since the phenomenological aspects of rewarding 
brain stimulation cannot be adequately examined at present, we are forced to analyze ESB within the traditional categories of motivational variables. It is traditionally assumed that the drive variable relates to changes in organismic states produced by deprivation conditions and/or strong stimulation, while the incentive variable relates to the nature of the reward and to its delivery. Although Gallistel's definition of drive (i.e., "... whatever produces changes in the direction and avidity of behavior in the absence of changes in the animal's knowledge of the reward contingencies") does in general account for this differentiation, it fails to specify the variables which contribute to drive, and also includes response directionality as a consequence of drive manipulations rather than as a consequence of incentive related stimuli as has traditionally been done.

It is our contention that the proper differentiation of drive and incentive variables is crucial for understanding the nature of performance for ESB. More importantly, the distinction between the two positions, induced "drive" vs reward-induced incentive, can be better appreciated by the different predictions which they generate. Possibly the most difficult data for the drive-decay position to assimilate was presented by Panksepp \& Trowill (1968) in their demonstration that, under special training conditions, animals show the highest extinction responding after a period of no reward rather than immediately after a period of reinforcement. A forthcoming review summarizes other data difficult for the drive decay position to explain (Trowill, Panksepp, \& Gandelman, in press).

The drive-decay position has led to interesting predictions (e.g., extinction without responding) which were not previously noted in the behavior of animals working for food or water. The incentive position does not deny such phenomena; rather, it suggests that they are transient phenomena associated with responding for strong rewards, of any type, under the conditions of nondeprivation (Panksepp \& Trowill, 1967).

Gallistel also cites the provocative results of Deutsch which purport to demonstrate parallel, but separate, fiber pathways in the brain which control "reward" and "drive." While we do not question Deutsch's results, we do question his interpretation. There may be many functional purposes served by parallel-running pathways with differing fiber refractory times. For example, such parallel paths could represent centripetal (sensory-afferents) and centrifugal (sensory-efferents) fiber bundles. The relevance of Deutsch's elegant experiments thus seems unclear.

Gallistel also takes issue with our citation of Scott's data (1967). As far as we can see, Scott's data only serve to weaken the drive-decay hypothesis. The crucial factor in discussing Scott's data in relation to our own is not in the fact that our animals still showed overnight decrements, which is common with conventional rewards, especially under low-deprivation conditions, but that with Scott's procedure (i.e., much practice with long ITIs), the decremental effect of even 24-h ITIs could be overcome. In fact, Scott felt that the running speed of his animals after 24-h intervals was comparable to those reported for massed trials. During the last postshift days our animals also showed no differences in running speed despite the differences in ITIs. A rigorous drive-decay explanation should, we believe, predict that after a $24-\mathrm{h}$ interval Ss should show little motivation to perform at all, no matter what the training conditions. At the very least, the initial trials of each day should always show an enormous performance decrement and should never attain the speeds emitted during massed practice.

\section{REFERENCES}

DEUTSCH, J. A., \& HOWARTH, C. I. Some tests of a theory of intracranial self-stimulation. Psychological Review, 1963, 70, 444-460.
GALLISTEL, C. R. Electrical self-stimulation and its theoretical implications. Psychological Bulletin, 1964, 61, 23-34.

GALLISTEL, C. R. Intracranial stimulation and natural reward: Differential effects of trial spacing. Psychonomic Science, 1967, 9, 167-168.

GALLISTEL, C. R. Comments on Panksepp et al. Psychonomic Science, 1969, 00, 000-000.

PANKSEPP, J., GANDELMAN, R., \& TROWILL, $J$. The effect of intertrial interval on running performance for ESB. Psychonomic Science, $1968,13,135-136$.

PANKSEPP, J., \& TROWILL, J. A. Intraoral self-injection: II. The simulation of selfstimulation phenomenon with a conventional reward. Psychonomic Science, 1967, 9, 407-408.

PANKSEPP, J., \& TROWILL, J. A. Extinction following intracranial reward: Frustration or drive decay? Psychonomic Science, 1968, 12, 173-174.

PANKSEPP, J., \& TROWILL, J. A. Positive and negative contrast effects with hypothalamic reward. Physiology and Behavior, 1969, 4, 173-175.

SCOTT, J. W. Brain stimulation reinforcement with distributed practice: Effects of electrode locus, previous experience and stimulus intensity. Journal of Comparative \& Physiological Psychology, 1967,63, 175-183.

TROWILL, J. A., PANKSEPP, J., \& GANDELMAN, $R$. An incentive model of rewarding brain stimulation. Psychological Review, in press.

\section{Drug effects on mouse exploratory behavior'}

\section{HAROLD G. WAKELEY and DENNIS O'SULLIVAN, IIT Research Institute, Chicago, Ill. 60616}

Ditran, atropine sulfate, d-amphetamine, chlorpromazine, or isotonic saline were administered intraperitoneally to $B D F_{1}$ mice prior to measurement of exploratory activity. D-amphetamine and chlorpromazine decreased exploration, whereas the anticholinergic compounds Ditran and atropine sulfate increased exploratory activity.

Primary screening tests that differentiate the effects of anticholinergic drugs from the effects of general stimulant or depressant drugs on animals are of value in assaying compounds for potential psychotropic effects in humans. Screening methods used for anticholinergic compounds have included measures of anorexia (Cohen, 1965) or changes in spontaneous activity (Lipman et al, 1963). Conventional locomotor or spontaneous activity tests ascribe increased activity to both anticholinergic compounds and stimulants such as amphetamine (Tripod et al, 1954). Observations of anticholinergic drug-induced changes in responsiveness to stimuli indicated that measurement of exploratory activity rather than locomotor activity might differentiate anticholinergic compounds from stimulants (Abood et al, 1959).

Carlton (1963) has suggested that a cholinergic system in the brain antagonizes a catecholamine system than activates behavior. The cholinergic antagonism is selective and proportional to the degree that behavior is unrewarded. According to this hypothesis, anticholinergic compounds should increase responsiveness to stimuli. Compounds that affect the catecholamine system, such as d-amphetamine, should shift the activity level, though not necessarily the response to specific stimuli.

An appropriate test should differentiate between the change in responsiveness to stimuli induced by anticholinergics and the change in activation level induced by compounds affecting the catecholamine system. The exploratory behavior test of Boissier \& Simon (1964) was selected. Exploration is defined as the performance of a quantifiable investigatory behavior pattern in response to specific stimuli, as contrasted with generalized locomotor activity.

To determine whether anticholinergic 